\title{
An Extended EDAS Method for Multicriteria Decision-Making Based on Multivalued Neutrosophic Sets
}

\author{
Lili Han $\mathbb{D}^{1}$ and Cuiping Wei $\mathbb{D}^{2,3}$ \\ ${ }^{1}$ College of Management, Qufu Normal University, Rizhao 276826, China \\ ${ }^{2}$ School of Mathematical Science, Yangzhou University, Yangzhou 225002, China \\ ${ }^{3}$ School of Information Science and Engineering, Qufu Normal University, Rizhao 276826, China \\ Correspondence should be addressed to Cuiping Wei; cpwei@yzu.edu.cn
}

Received 22 August 2019; Revised 6 January 2020; Accepted 22 January 2020; Published 24 February 2020

Academic Editor: Roberto Natella

Copyright ( 92020 Lili Han and Cuiping Wei. This is an open access article distributed under the Creative Commons Attribution License, which permits unrestricted use, distribution, and reproduction in any medium, provided the original work is properly cited.

\begin{abstract}
Neutrosophic set (NS) is a generalization of intuitionistic fuzzy set (IFS). It depicts not only the incomplete information but also the indeterminate information and inconsistent information which exist commonly in belief systems. In this paper, the evaluation based on distance from average solution (EDAS) method is extended to handle multicriteria decision-making problems with multivalued neutrosophic numbers (MVNNs). The average solution under all the criteria is calculated by the proposed convex weighted average operator of MVNNs. Then, the positive distance and the negative distance from each solution to the average solution are calculated, and the comprehensive evaluations of alternatives are obtained by integrating two kinds of distance values to get the ranking result. Finally, the rationality and efficiency of the proposed method are shown by the parameter analysis and comparisons with some existing methods.
\end{abstract}

\section{Introduction}

Zadeh $[1,2]$ proposed the fuzzy set (FS) theory, which describes the uncertain information in the form of membership function. In some cases, it is difficult for the membership function to give a complete description of the decision information alone. So, Atanassov [3, 4] proposed the concept of intuitionistic fuzzy sets (IFSs) on this basis of FSs by adding a nonmembership function. Torra and Narukawa [5,6] proposed hesitant fuzzy sets (HFSs) to describe the situation in which the expert is hesitant when giving evaluations. IFSs and HFSs have attracted a lot of researchers' attention and obtained many research results, which have been widely used to solve problems of multicriteria decision-making (MCDM) in engineering and management [7-9].

FSs theory has been well developed, but it cannot distinguish between abandonment and uncertain information that are widely available in the real world. For example, in a decision-making process, suppose there are
10 decision makers, of which 4 decision makers agree, 3 decision makers disagree, 2 decision makers are uncertain, and 1 decision maker gives up. For this case, traditional fuzzy sets cannot accurately describe. To handle this situation, Smarandache $[10,11]$ proposed the concept of neutrosophic set (NS) by adding an independent indeterminacy-membership degree. For the abovementioned situation, the information can be expressed by a NS as $\{x(0.4,0.3,0.2)\}$. NSs were originally introduced from a philosophical perspective, and it is difficult to be applied in solving practical problems. Considering this situation, some special cases of NS are proposed, such as single-valued neutrosophic sets (SVNSs) and interval neutrosophic sets (INSs) [12-14]. In recent years, many research results about SVNSs and INSs have been made involving average operator, NWBM operator, generalized hybrid weight average operator, crossentropy, some similarity measures, etc., [15-23], which are widely used in solving decision-making problems. In addition, NS theory and related research results have been applied in 
other fields, for example, graph theory [24-29], cluster analysis [30-32], image processing [33-35], medical diagnosis and treatment systems [36-38], social management [39-41], and artificial intelligence [42].

However, in some decision-making environment, multiple different discrete values are needed to represent decision information, rather than using specific or interval values. Under these circumstances, Wang and $\mathrm{Li}$ [43] proposed the definition of multivalued neutrosophic sets (MVNSs) and developed the TODIM method to solve the multicriteria decision-making (MCDM) with MVNSs. Liu [44] proposed some aggregation operators of MVNSs and applied them in decision-making problems. Peng and Wang [45] introduced a comparison method and some aggregation operators of MVNSs. Peng et al. [46, 47] also developed a multivalued neutrosophic qualitative flexible approach based on likelihood and an extended ELECTRE approach with multivalued neutrosophic information. Ji et al. [48] proposed a projection-based TODIM method under multivalued neutrosophic environments.

Ghorabaee et al. [49] proposed a new method called evaluation on distance from average solution (EDAS) for multicriteria inventory classification. This method ranks the alternatives by calculating the positive distance and negative distance between each alternative and the average alternative. The good performance of the EDAS method in solving the inventory classification problems and MCDM problems was also shown in [49]. EDAS is a simple and effective method for MCDM, but it can only deal with decision information described by numerical values.

Motivated by these ideas, in this paper, we propose an extended EDAS method to solve the MCDM problem, which is described by MVNSs. We revise the aggregation method of the positive distance and negative distance in [49] and permit the decision makers to adjust their proportions according to their preferences in calculating the comprehensive evaluations of alternatives. So, the method is more flexible for decision makers in choosing the most appropriate solution. Moreover, compared with some existing methods, it needs fewer data preprocessing and calculation and is suitable to solve the decisionmaking problem with more criteria and alternatives. The proposed method also can be used to the situations in which the decision information is described by SVNSs, HFSs, or Dual HFSs.

This paper is constructed as follows. Section 2 introduces the basic concepts and operations of of MVNSs. In Section 3, the EDAS method for MCDM problem with MVNSs is proposed. Algorithm analysis and comparison analysis are made based on two numerical examples to illustrate the feasibility and effectiveness of the proposed method in Section 4. Finally, the conclusions are given in Section 5.

\section{Preliminaries}

2.1. Multivalued Neutrosophic Sets. In this section, we recall some definitions, operations, and properties regarding MVNSs, which will be used in the rest of the paper.
Definition 1 (see [14]). Let $X$ be a universal set and $x$ be a generic element in $X$. A single-valued neutrosophic set (SVNS) in $X$ is defined as

$$
A=\left\{\left\langle x\left(T_{A}(x), I_{A}(x), F_{A}(x)\right)\right\rangle|x \in X\rangle\right\},
$$

where $T_{A}$ is a truth-membership function, $I_{A}$ is a indeterminacy-membership function, and $F_{A}$ is a falsity-membership function. For each point $x \in X$, we have $T_{A}, I_{A}$, $F_{A} \in[0,1]$ and $0 \leq T_{A}+I_{A}+F_{A} \leq 1$.

Definition 2 (see [45]). Let $X$ be a universal set and $x$ be a generic element in $X$. A multivalued neutrosophic number set (MVNS) in $X$ is defined as

$$
A=\left\{x\left(\widetilde{T}_{A}(x), \widetilde{I}_{A}(x), \widetilde{F}_{A}(x)\right) \mid x \in X\right\},
$$

where $\widetilde{T}_{A}(x), \widetilde{I}_{A}(x)$, and $\widetilde{F}_{A}(x)$ are three sets of discrete real values in $[0,1]$, denoting the truth-membership degree, indeterminacy-membership degree, and falsity-membership degree, respectively, which satisfies $0 \leq \gamma, \eta, \xi \leq 1$, and $0 \leq$ $\gamma^{+}+\eta^{+}+\xi^{+} \leq 3$, where $\gamma \in \widetilde{T}_{A}(x), \eta \in \widetilde{I}_{A}(x)$, and $\xi \in \widetilde{F}_{A}(x)$ and $\gamma^{+}=\sup \widetilde{T}_{A}(x), \eta^{+}=\sup \widetilde{F}_{A}(x)$, and $\xi^{+}=\sup \widetilde{I}_{A}(x)$.

For convenience, $\left\langle\widetilde{T}_{A}(x), \widetilde{I}_{A}(x), \widetilde{F}_{A}(x)\right\rangle$ is called a multivalued neutrosophic number (MVNN), which is denoted as $A=\left\langle\widetilde{T}_{A}, \widetilde{I}_{A}, \widetilde{F}_{A}\right\rangle$.

MVNS is a generalized form of SVNS, hesitant fuzzy set, and dual hesitant fuzzy set. In fact, if $\widetilde{T}_{A}(x), \widetilde{I}_{A}(x)$, and $\widetilde{F}_{A}(x)$, for any $x \in X$, has only one value, respectively, then a MVNSs is reduced to a SVNS. If $\widetilde{I}_{A}(x)=\Phi$ for any $x \in X$, then a MVNS is reduced to a dual hesitant fuzzy set [50]; if $\widetilde{I}_{A}(x)=\widetilde{F}_{A}(x)=\Phi$ for any $x \in X$, a MVNS is reduced to a hesitant fuzzy set [5].

Example 1. Assume that $X=\left\{x_{1}, x_{2}, x_{3}, x_{4}, x_{5}, x_{6}\right\}$ is the universal set, where $x_{1}$ is the functionality, $x_{2}$ is the reliability, $x_{3}$ is the ease of use, $x_{4}$ is the efficiency, $x_{5}$ is the maintainability, $x_{6}$ is the portability of software, and the evaluations of a software under $x_{1}, x_{2}, x_{3}, x_{4}, x_{5}$, and $x_{6}$ are in $[0,1]$. Evaluation information about a software can be obtained from some experts in the field. Their opinions about the degree of "good," a degree of indeterminacy, and a degree of "poor" can be described by the following MVNS in $X$ :

$$
\begin{aligned}
A= & \left\{x_{1}(\{0.4,0.5\},\{0.2\},\{0.3\}), x_{2}(\{0.6,0.7\},\{0.2\},\{0.3\}),\right. \\
& x_{3}(\{0.5\},\{0.2,0.3\},\{0.3\}), x_{4}(\{0.7,0.8\},\{0.1\},\{0.2\}), \\
& \left.x_{5}(\{0.2\},\{0.5\},\{0.5,0.6\}), x_{6}(\{0.3\},\{0.4,0.5\},\{0.5\})\right\} .
\end{aligned}
$$

2.2. The Comparison Method and Operational Rules. In order to apply MVNNSs to solve practical problems, some comparison and operational rules are reviewed as follows.

For any two MVNNs, they can be compared by the method proposed by Peng et al. [45]. 
Definition 3. Let $A=\left\langle\widetilde{T}_{A}, \widetilde{I}_{A}, \widetilde{F}_{A}\right\rangle$ be a MVNN. Then, its score function $s(A)$, accuracy function $a(A)$, and certainty function $c(A)$ are defined as follows:

(1) $s(A)=\left(1 /\left(l_{\widetilde{T}_{A}} \cdot l_{\widetilde{I}_{A}} \cdot l_{\widetilde{F}_{A}}\right)\right) \sum_{\gamma_{i} \in \widetilde{T}_{A}, \eta_{j} \in \widetilde{I}_{A}, \xi_{k} \in \widetilde{F}_{A}}\left(\gamma_{i}+1-\right.$ $\left.\eta_{j}+1-\xi_{k}\right) / 3$

(2) $a(A)=\left(1 /\left(l_{\widetilde{T}_{A}} \cdot l_{\widetilde{F}_{A}}\right)\right) \sum_{\gamma_{i} \in \widetilde{T}_{A}, \xi_{k} \in \widetilde{F}_{A}}\left(\gamma_{i}-\xi_{k}\right)$

(3) $c(A)=\left(1 / l_{\widetilde{T}_{A}}\right) \sum_{\gamma_{i} \in \widetilde{T}_{A}} \gamma_{i}$

where $\gamma_{i} \in \widetilde{T}_{A}, \eta_{j} \in \widetilde{I}_{A}, \xi_{k} \in \widetilde{F}_{A}$, and $l_{\widetilde{T}_{A}}, l_{\widetilde{I}_{A}}$, and $l_{\widetilde{F}_{A}}$ are the element numbers of $\widetilde{T}_{A}, \widetilde{I}_{A}$, and $\widetilde{F}_{A}$, respectively.

Definition 4 (see [45]). Let $A$ and $B$ be two MVNNs. Then, their comparison rules are defined as follows:

(1) If $s(A)>s(B)$, then $A>B$

(2) If $s(A)=s(B)$ and $a(A)>a(B)$, then $A>B$

(3) If $s(A)=s(B), a(A)=a(B)$ and $c(A)>c(B)$, then $A>B$

(4) If $s(A)=s(B), a(A)=a(B)$ and $c(A)=c(B)$, then $A \sim B$

Definition 5 (see [45]). Let $A=\left\langle\widetilde{T}_{A}, \widetilde{I}_{A}, \widetilde{F}_{A}\right\rangle$ and $B=$ $\left\langle\widetilde{T}_{B}, \widetilde{I}_{B}, \widetilde{F}_{B}\right\rangle$ be any two MVNNs. The operations on MVNNs are defined by

(i) $A \oplus B=\left\langle\cup_{\gamma_{A} \in \widetilde{T}_{A}, \gamma_{B} \in \widetilde{T}_{B}}\left\{\gamma_{A}+\gamma_{B}-\gamma_{A} \cdot \gamma_{B}\right\}\right.$, $\left.\cup_{\eta_{A} \in \widetilde{T}_{A}, \eta_{B} \in \widetilde{T}_{B}}\left\{\eta_{A} \cdot \eta_{B}\right\}, \cup_{\xi_{A} \in \widetilde{T}_{A}, \xi_{B} \in \widetilde{T}_{B}}\left\{\xi_{A} \cdot \xi_{B}\right\}\right\rangle$

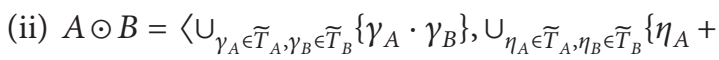
$\left.\left.\eta_{B}-\eta_{A} \cdot \eta_{B}\right\}, \cup_{\xi_{A} \in \widetilde{T}_{A}, \xi_{B} \in \widetilde{T}_{B}}\left\{\xi_{A}+\xi_{B}-\xi_{A} \cdot \xi_{B}\right\}\right\rangle$

(iii) $\lambda A=\left\langle\cup_{\gamma_{A} \in \widetilde{T}_{A}}\left\{1-\left(1-\gamma_{A}\right)^{\lambda}\right\}, \cup_{\eta_{A} \in \widetilde{I}_{A}}\left\{\left(\eta_{A}\right)^{\lambda}\right\}\right.$, $\left.\cup_{\xi_{A} \in \widetilde{F}_{A}}\left\{\left(\xi_{A}\right)^{\lambda}\right\}\right\rangle, \lambda>0$ (iv) $A^{\lambda}=\left\langle\cup_{\gamma_{A} \in \widetilde{T}_{A}}\left\{\left(\gamma_{A}\right)^{\lambda}\right\}, \cup_{\eta_{A} \in \widetilde{I}_{A}}\left\{1-\left(1-\eta_{A}\right)^{\lambda}\right\}, \quad \cup_{\xi_{A} \in \widetilde{F}_{A}}\right.$ $\left.\left\{1-\left(1-\xi_{A}\right)^{\lambda}\right\}\right\rangle, \lambda>0$

Let $A=\left\langle\widetilde{T}_{A}, \widetilde{I}_{A}, \widetilde{F}_{A}\right\rangle$ and $B=\left\langle\widetilde{T}_{B}, \widetilde{I}_{B}, \widetilde{F}_{B}\right\rangle$ be two MVNNs. Then, the following properties are easily obtained from the abovementioned operations:

(i) $A \oplus B=B \oplus A$

(ii) $A \odot B=B \odot A$

(iii) $\lambda(A \oplus B)=\lambda A \oplus \lambda B, \lambda>0$

(iv) $(A \odot B)^{\lambda}=A^{\lambda} \odot B^{\lambda}, \lambda>0$

(v) $\left(\lambda_{1}+\lambda_{2}\right) A=\lambda_{1} A \oplus \lambda_{2} A, \lambda>0$

(vi) $A^{\lambda_{1}+\lambda_{2}}=A^{\lambda_{1}} \odot A^{\lambda_{2}}, \lambda_{1}, \lambda_{2}>0$

\section{Aggregation Operators and EDAS Method with MVNNSs}

3.1. Aggregation Operators. In this section, we define the weighted average operators for MVNNS by using the idea of the convex combination [51].

Definition 6. Let $A=\left\langle\widetilde{T}_{A}, \widetilde{I}_{A}, \widetilde{F}_{A}\right\rangle$ and $B=\left\langle\widetilde{T}_{B}, \widetilde{I}_{B}, \widetilde{F}_{B}\right\rangle$ be any two MVNNs. The convex combination of $A$ and $B$ can be defined as follows:

$$
C^{2}\left(\omega_{1}, A, \omega_{2}, B\right)=\omega_{1} A \oplus \omega_{2} B,
$$

where $\omega_{i} \geq 0(i=1,2)$ and $\omega_{1}+\omega_{2}=1$.

Definition 7. Let $X=\left\{x_{i} \mid x_{i}=\left\langle\widetilde{T}_{x_{i}}, \widetilde{I}_{x_{i}}, \widetilde{F}_{x_{i}}\right\rangle, i=1,2, \ldots, n\right\}$ be a collection of MVNNs. We called

$$
\operatorname{MVNCWA}\left(x_{1}, x_{2}, \ldots, x_{n}\right)=C^{n}\left(\omega_{i}, x_{i}\right)=\omega_{1} x_{1} \oplus\left(1-\omega_{1}\right) C^{n-1}\left(\frac{\omega_{k}}{\sum_{k=2}^{n} \omega_{k}}, x_{k}\right)
$$

A multivalued neutrosophic extended weighted average (MVNCWA) operator, where $\omega_{i}$ is the weight vector of $x_{i}$ and $\omega_{i} \geq 0(i=1,2, \ldots, n), \sum_{i=1}^{n} \omega_{i}=1$.
We consider a special case of Definition 7. If $\omega_{i}=(1 / n)(i=1,2, \ldots, n)$, then the MVNCWA operator reduces to the arithmetic average operator with the following form:

$$
\operatorname{MVNCWA}\left(x_{1}, x_{2}, \ldots, x_{n}\right)=C^{n}\left(\omega_{i}, x_{i}\right)=C^{n}\left(\frac{1}{n}, x_{i}\right)=\frac{1}{n} x_{1} \oplus\left(1-\frac{1}{n}\right) C^{n-1}\left(\frac{1}{n-1}, x_{k}\right)
$$

3.2. An Extended EDAS Method with MVNNS. In this section, we present an algorithm to handle MCDM problems with MVNNs based on the EDAS method proposed by Ghorabaee et al. [49]. In this method, first, calculate the average alternative with the existing alternative. Next, calculate the positive distance and negative distance of each alternative relative to the average alternative. Then, the comprehensive evaluations of alternatives are obtained by considering the influence of positive and negative distance on an average. Based on this idea, we propose an extended EDAS method to deal with decision information described by MVNNs.

The multiattribute decision-making problems based on MVNNs can be considered as follows. Let $A=\left\{A_{1}, A_{2}, \ldots\right.$, $\left.A_{m}\right\}$ be the set of alternatives, $C=\left\{c_{1}, c_{2}, \ldots, c_{n}\right\}$ be the set of criteria, $B$ denote the index set of beneficial criteria, and $N$ 
denote the index set of cost criteria. Let $\omega=\left\{\omega_{1}, \omega_{2}, \ldots, \omega_{m}\right\}$ with $\omega_{i} \geq 0, \sum_{i=1}^{m} \omega_{i}=1$ be the weight vector of the creteria. Suppose that $R=\left[R_{i j}\right]_{m \times n}$ is the decision matrix, where $R_{i j}=$ $\left\langle\widetilde{T}_{i j}, \widetilde{I}_{i j}, \widetilde{F}_{i j}\right\rangle$ is a MVNN satisfying $\widetilde{T}_{i j}, \widetilde{I}_{i j}, \widetilde{F}_{i j} \in[0,1]$, and describes the evaluation information of the alternative $A_{i}$ with respect to the criterion $c_{j}$.

Here, we give the basic idea of the extended EDAS decision-making method based on MVNNs. Firstly, the average alternative is calculated by the proposed weighted average operator given in Definition 7. Next, the score function of MVNN proposed in [45] is comonly used to calculate the dominance of the alternative, and the positive distance matrix and negative distance matrix are constructed by calculating the positive and negative distance between each alternative and the average alternative. Finally, the weighted average of the normalized positive and negative distance is used to obtain the comprehensive evaluation value of each alternative.

The concrete algorithm of EDAS for MCDM with MVNNs is described as follows.

Step 1: construct the decision matrix $R=\left[R_{i j}\right]_{m \times n}$, whose elements are described by the multivalued neutrosophic numbers.

Step 2: aggregate the evaluations of all the alternatives under each criterion by equation (6) and get the average alternative $\mathrm{AV}=\left(\mathrm{AV}_{1}, \mathrm{AV}_{2}, \ldots, \mathrm{AV}_{i}, \ldots, \mathrm{AV}_{n}\right)$, where

$$
\begin{aligned}
\mathrm{AV}_{i} & =\operatorname{MVNCWA}\left(R_{1 i}, R_{2 i}, \ldots, R_{m i}\right) \\
& =C^{m}\left(\frac{1}{n}, R_{k i} \mid k=1,2, \ldots, m\right) \\
& =\frac{1}{n} R_{1 i} \oplus\left(1-\frac{1}{n}\right) C^{m-1}\left(\frac{1}{n-1}, R_{k i}\right) .
\end{aligned}
$$

Step 3: calculate the positive distance matrix PDA = $\left(\mathrm{PDA}_{i j}\right)_{m \times n}$ and negative distance matrix $\mathrm{NDA}=$ $\left(\mathrm{NDA}_{i j}\right)_{m \times n}$ of each alternative from the average alternative according to equations (8) and (9):

$$
\begin{gathered}
\mathrm{PDA}_{i j}= \begin{cases}\frac{\max \left(0,\left(s\left(R_{i j}\right)-s\left(\mathrm{AV}_{j}\right)\right)\right)}{s\left(\mathrm{AV}_{j}\right)}, & j \in B, \\
\frac{\max \left(0,\left(s\left(\mathrm{AV}_{j}\right)-s\left(R_{i j}\right)\right)\right)}{s\left(\mathrm{AV}_{j}\right)}, & j \in N,\end{cases} \\
\mathrm{NDA}_{i j}= \begin{cases}\frac{\max \left(0,\left(s\left(\mathrm{AV}_{j}\right)-s\left(R_{i j}\right)\right)\right)}{s\left(\mathrm{AV}_{j}\right)}, & j \in B, \\
\frac{\max \left(0,\left(s\left(R_{i j}\right)-s\left(\mathrm{AV}_{j}\right)\right)\right)}{s\left(\mathrm{AV}_{j}\right)}, & j \in N .\end{cases}
\end{gathered}
$$

Remark 1. If $s\left(\mathrm{AV}_{j}\right)=s\left(\mathrm{R}_{i j}\right)$ and $a\left(\mathrm{AV}_{j}\right) \neq a\left(\mathrm{R}_{i j}\right)$, then replace score function with accuracy function in equations (8) and (9); if $s\left(\mathrm{AV}_{j}\right)=s\left(\mathrm{R}_{i j}\right)$ and $a\left(\mathrm{AV}_{j}\right)=$
$a\left(\mathrm{R}_{i j}\right)$, then replace score function with certainty function in equations (8) and (9).

Step 4: calculate the positive distance and the negative distance of each alternative $A_{i}(i=1, \ldots, m)$, denoted as $\mathrm{PD}_{i}$ and $\mathrm{ND}_{i}$, respectively, by

$$
\begin{aligned}
\mathrm{PD}_{i} & =\sum_{j=1}^{n} w_{j} \mathrm{PDA}_{i j}, \\
\mathrm{ND}_{i} & =\sum_{j=1}^{n} w_{j} \mathrm{NDA}_{i j} .
\end{aligned}
$$

Step 5: the normalized values of PD and ND (NPD and NND) for all alternatives can be calculated by

$$
\begin{aligned}
\mathrm{NPD}_{i} & =\frac{\mathrm{PD}_{i}}{\max _{i}(\mathrm{PD}(i))}, \\
\mathrm{NND}_{i} & =\frac{\mathrm{ND}_{i}}{\max _{i}(\mathrm{ND}(i))} .
\end{aligned}
$$

Step 6: the comprehensive evaluation of each alternative $\operatorname{AS}_{i}(i=1,2, \ldots, m)$ can be calculated by

$$
\mathrm{AS}_{i}=\lambda \mathrm{NPD}_{i}+(1-\lambda)\left(1-\mathrm{NND}_{i}\right), \quad \lambda \in[0,1],
$$

where the decision makers can adjust the value of $\lambda$ according to their preference for positive distance and negative distance.

Especially, when the preference of the decision-maker is neutral and taken $\lambda=(1 / 2),(12)$ is simplified to

$$
\mathrm{AS}_{i}=\frac{1}{2}\left(\mathrm{NPD}_{i}+\left(1-\mathrm{NND}_{i}\right)\right)
$$

Step 7: rank the alternatives according to the comprehensive evaluation value $\operatorname{AS}_{i}(i=1,2, \ldots, m)$ and then choose the satisfactory scheme.

\section{Illustrative Example}

4.1. An Illustrative Example of the EDAS Method. Based on the multicriteria decision-making problem used in [22, 43], the feasibility and effectiveness of the proposed method are analyzed. The multiattribute decision-making problem is described as follows. An investment company needs to choose appropriate investment projects so that to get rich reward. There are four possible investment options: (1) $A_{1}$ : automobile company; (2) $A_{2}$ : food company; (3) $A_{3}$ : computer company; (4) $A_{4}$ : military industrial enterprise. In order to select appropriate investment project and obtain satisfied investment returns, the investment company should consider the enterprise performance of the investment project. The enterprise performance is a comprehensive reflection of its profitability, operation, risk management, debt repayment, and social responsibility in the company's production and operation process. The ability of enterprises to fulfill their social responsibilities is the basis of their long-term existence and development. The social 
responsibility ability of enterprises is manifested in many aspects. We choose relatively important environmental factors. After discussion by the company's development committee, the following three criteria are considered in the selection of investment options: (1) $C_{1}$ : risk control capability; (2) $C_{2}$ : growth factors; (3) $C_{3}$ : environmental impact factors, where $C_{1}$ and $C_{2}$ are benefit criteria and $C_{3}$ is a cost criterion. In this problem, the weight vector of criteria is given by $w=(0.2,0.25,0.55)$.
Next, we apply the algorithm proposed in Section 3.2 to solve this decision-making problem.

Step 1: the decision matrix described by the multivalued neutrosophic sets is given by the decision maker, which is shown in Table 1:

Step 2: calculate the average solution $A V=\left(A V_{1}\right.$, $A V_{2}, A V_{3}$ ) according to equation (6), where

$$
\begin{aligned}
\mathrm{AV}_{1} & =\frac{1}{4} R_{11} \oplus\left(1-\frac{1}{4}\right)\left(\frac{1}{3} R_{21} \oplus\left(1-\frac{1}{3}\right)\left(\frac{1}{2} R_{31} \oplus\left(1-\frac{1}{2}\right) R_{41}\right)\right) \\
& =\frac{1}{4}\left\langle\bigcup_{\gamma_{R_{k 1}} \in \widetilde{T}_{R_{k 1}}}\left\{1-\prod_{k=1}^{3}\left(1-\gamma_{R_{k 1}}\right)\right\}, \underset{\eta_{R_{k 1}} \in \widetilde{I}_{R_{k 1}}}{\bigcup}\left\{\prod_{k=1}^{3} \eta_{R_{k 1}}\right\}, \bigcup_{\xi_{R_{k 1}} \in \widetilde{F}_{R_{k 1}}}^{\bigcup}\left\{\prod_{k=1}^{3} \xi_{R_{k 1}}\right\}\right\rangle .
\end{aligned}
$$

So, by Definition 3, the score function value of $A V_{1}$ can be obtained as

$$
s\left(\mathrm{AV}_{1}\right)=0.7234 .
$$

In a similar way, we can get $s\left(\mathrm{AV}_{2}\right)=0.7127$, $s\left(\mathrm{AV}_{3}\right)=0.6489$.

Step 3: calculate the positive distance matrix PDA and negative distance matrix NDA by equations (8) and (9), where $B=\left\{C_{1}, C_{2}\right\}$ and $N=\left\{C_{3}\right\}$ :

$$
\begin{aligned}
\mathrm{PDA} & =\left[\begin{array}{ccc}
0 & 0 & 0.2295 \\
0.0368 & 0.0758 & 0 \\
0 & 0 & 0 \\
0.1290 & 0.0758 & 0.0240
\end{array}\right], \\
\mathrm{NDA} & =\left[\begin{array}{ccc}
0.1014 & 0.1347 & 0 \\
0 & 0 & 0.1044 \\
0.1475 & 0.0646 & 0.0530 \\
0 & 0 & 0
\end{array}\right] .
\end{aligned}
$$

Step 4: according to equation (10), the positive distance $\mathrm{PD}_{i}$ and the negative distance $\mathrm{ND}_{i}(i=1,2,3,4)$ of each alternative are calculated as follows:

$$
\begin{aligned}
\mathrm{PD}_{1} & =0.0918, \\
\mathrm{PD}_{2} & =0.0318, \\
\mathrm{PD}_{3} & =0, \\
\mathrm{PD}_{4} & =0.0737, \\
\mathrm{ND}_{1} & =0.0692, \\
\mathrm{ND}_{2} & =0.0417, \\
\mathrm{ND}_{3} & =0.0890, \\
\mathrm{ND}_{4} & =0 .
\end{aligned}
$$

Step 5: the normalized values $\mathrm{NPD}_{i}$ and $\mathrm{NND}_{i}$ for all alternatives are shown as follows:

$$
\begin{aligned}
\mathrm{NPD}_{1} & =1.0000, \\
\mathrm{NPD}_{2} & =0.2048, \\
\mathrm{NPD}_{3} & =0, \\
\mathrm{NPD}_{4} & =0.4592, \\
\mathrm{NND}_{1} & =0.7215, \\
\mathrm{NND}_{2} & =0.7675, \\
\mathrm{NND}_{3} & =1.0000, \\
\mathrm{NND}_{4} & =0 .
\end{aligned}
$$

Step 6: when $\lambda=0.5$ is taken in equation (12), the comprehensive evaluations $\mathrm{AS}_{i}(i=1,2,3,4)$ of alternatives can be obtained:

$$
\begin{aligned}
& \mathrm{AS}_{1}=0.6393, \\
& \mathrm{AS}_{2}=0.2204, \\
& \mathrm{AS}_{3}=0, \\
& \mathrm{AS}_{4}=0.7296 .
\end{aligned}
$$

Step 7: the ranking of the four projects is $A_{4}>A_{1}>$ $A_{2}>A_{3}$. Obviously, $A_{4}$ is the best alternative.

4.2. Algorithmic Analysis. In Step 6 of the proposed algorithm, decision makers can adjust the value of $\lambda$ according to their preference for positive distance and negative distance. In order to study the influence of different parameter values on ranking results, we take different values of $\lambda$ to calculate the ranking results of the alternatives, which are shown in Table 2.

From Table 2, it can be seen that the ranking results are consistent for $\lambda \leq 0.5$ and $\lambda>0.5$, respectively. On the other hand, the ranking for $\lambda \leq 0.5$ is slightly different from that for $\lambda>0.5$. When $\lambda \leq 0.5$, the best scheme is $A_{4}$ and the worst 
TABle 1: Decision-making matrix $R$.

\begin{tabular}{llcc}
\hline & \multicolumn{1}{c}{$c_{1}$} & $c_{2}$ & $c_{3}$ \\
\hline$A_{1}$ & $\{\{0.4,0.5\},\{0.2\},\{0.3\}\}$ & $\{\{0.4\},\{0.2,0.3\},\{0.3\}\}$ & $\{\{0.2\},\{0.2\},\{0.5\}\}$ \\
$A_{2}$ & $\{\{0.6\},\{0.1,0.2\},\{0.2\}\}$ & $\{\{0.6\},\{0.1\},\{0.2\}\}$ & $\{\{0.5\},\{0.2\},\{0.1,0.2\}\}$ \\
$A_{3}$ & $\{\{0.3,0.4\},\{0.2\},\{0.3\}\}$ & $\{\{0.5\},\{0.2\},\{0.3\}\}$ & $\{\{0.5\},\{0.2,0.3\},\{0.2\}\}$ \\
$A_{4}$ & $\{\{0.7\},\{0.1,0.2\},\{0.1\}\}$ & $\{\{0.6\},\{0.1\},\{0.2\}\}$ & $\{\{0.3\},\{0.3\},\{0.2\}\})$ \\
\hline
\end{tabular}

TABle 2: Compared results utilizing different values of $\lambda$.

\begin{tabular}{|c|c|c|c|}
\hline & The ranking & The best alternative & The worst alternative \\
\hline$\lambda=0.1$ & $A_{4}>A_{1}>A_{2}>A_{3}$ & $A_{4}$ & $A_{3}$ \\
\hline$\lambda=0.2$ & $A_{4}>A_{1}>A_{2}>A_{3}$ & $A_{4}$ & $A_{3}$ \\
\hline$\lambda=0.3$ & $A_{4}>A_{1}>A_{2}>A_{3}$ & $A_{4}$ & $A_{3}$ \\
\hline$\lambda=0.4$ & $A_{4}>A_{1}>A_{2}>A_{3}$ & $A_{4}$ & $A_{3}$ \\
\hline$\lambda=0.5$ & $A_{4}>A_{4}>A_{2}>A_{3}$ & $A_{4}$ & $A_{3}$ \\
\hline$\lambda=0.6$ & $A_{1}>A_{4}>A_{2}>A_{3}$ & $A_{1}^{4}$ & $A_{3}$ \\
\hline$\lambda=0.7$ & $A_{1}>A_{4}>A_{2}>A_{3}$ & $A_{1}$ & $A_{3}$ \\
\hline$\lambda=0.8$ & $A_{1}>A_{4}>A_{2}>A_{3}$ & $A_{1}$ & $A_{3}$ \\
\hline$\lambda=0.9$ & $A_{1}>A_{4}>A_{2}>A_{3}$ & $A_{1}$ & $A_{3}$ \\
\hline
\end{tabular}

scheme is $A_{3}$; when $\lambda>0.5$, the best scheme is $A_{1}$ and the worst scheme is $A_{3}$. The best one is $A_{4}$ or $A_{1}$ depending on the value of $\lambda$, while the worst one is always $A_{3}$ for different values of $\lambda$. So, the ranking results are stable with the different values of $\lambda$.

Compared with the original EDAS in [49] that fixed the value of $\lambda$ to 0.5 , the proposed method allows parameter $\lambda$ from 0 to 1 and gives full consideration of the preference of decision makers for loss and gain to the average solution by adjusting the values of $\lambda$. When $\lambda<0.5$, the loss of the alternative relative to the average solution is magnified. Otherwise, more attention is paid to the gain of the scheme over the average solution. The decision makers can adjust the value of $\lambda$ according to their preference so as to solve the actual decision-making problem with a perfect choice.

\subsection{Comparison Analysis}

4.3.1. Comparison with the Methods for MVNSs. There are other methods for the multicriteria decision problem with MVNSs, for example, the MVNWA and MVNGA operators proposed by Peng and Wang [45] are used to solve MCDM with MVNSs. The TODIM method was put forward by Wang and $\mathrm{Li}$ [43] to deal with decision information described with MVNSs. In order to verify the effectiveness of the proposed algorithm, we made a comparative analysis with these methods by using the case in Section 4.1. The calculation results of the three methods are shown in Table 3.

From Table 3, it can be seen that the best alternative is $A_{4}$ for all the methods and the worst one is $A_{2}$ for the method using the MVNGA operator in [45], while the worst one is $A_{3}$ for all other methods. The ranking result of our proposed method taking $\lambda \leq 0.5$ is the same as that of the method in [43] and the method using the MVNWA operator in [45].

The TODIM method [43] can take into account the decision makers' psychological behavior and obtain more decision information by calculating the dominance of any two alternatives under each criterion. But when there are more alternatives in the MCDM problems, we need to deal with the dominance degree matrices with larger order. By the methods in [45], different aggregation operators can be used to deal with the different relationships of the aggregated arguments. But the number of operations and the sizes of the results will exponentially increase if more MVNNs are involved in the operations. Therefore, it is not suitable to deal with the MCDM problems with large number of criteria. Compared with these methods, the proposed method has the advantages of the simplicity and less calculation process and is more suitable to deal with MVNNs under large number of criteria.

4.3.2. Comparison with the Methods for SVNSs. In this section, the proposed method is compared with the two methods of Ye [21] and Chu and Liu [17] for simplified neutrosophic information.

The proposed method is also suitable for MCDM with SVNSs. In this situation, Ye [21] proposed the crossentropy of SVNSs and use it to solve MCDM problem, and Chi and Liu [17] proposed an expanded TOPSIS method to deal with interval neutrosophic information, which is suitable for simplified neutrosophic information since a numerical number can be seen an interval. In order to facilitate the comparative analysis, we cite the same illustrative example in [21] to compare the three methods.

The problem to be solved is to choose the best supplier based on the suppliers' core competitiveness. Suppose that there are four suppliers $A_{1}, A_{2}, A_{3}$, and $A_{4}$ whose core competencies are evaluated by the following four criteria $C_{1}, C_{2}, C_{3}$, and $C_{4}$ : (1) the level of technology innovation $C_{1}$, (2) the control ability of flow $C_{2}$, (3) the ability of management $C_{3}$, and (4) the level of service $C_{4}$. Suppose the weight vector of the four criteria is $w=(0.3,0.25,0.25,0.2)$. The decision matrix described by the single-valued 
TABLE 3: Ranking results by different methods.

\begin{tabular}{|c|c|c|c|}
\hline The method & The ranking & The best & The worst \\
\hline MVNWA & $A_{4}>A_{1}>A_{2}>A_{3}$ & $A_{4}$ & $A_{3}$ \\
\hline MVNGA & $A_{4}>A_{1}>A_{3}>A_{2}$ & $A_{4}$ & $A_{2}$ \\
\hline $\operatorname{TODIM}(\theta=1)$ & $A_{4}>A_{1}>A_{2}>A_{3}$ & $A_{4}$ & $A_{3}$ \\
\hline The proposed method $(\lambda \leq 0.5)$ & $A_{4}>A_{1}>A_{2}>A_{3}$ & $A_{4}$ & $A_{3}$ \\
\hline
\end{tabular}

TABLE 4: Decision-making matrix.

\begin{tabular}{lccrr}
\hline & $C_{1}$ & $C_{2}$ & $C_{3}$ & $C_{4}$ \\
\hline$A_{1}$ & $\{0.5,0.1,0.3\}$ & $\{0.5,0.1,0.4\}$ & $\{0.7,0.1,0.2\}$ & $\{0.3,0.2,0.1\}$ \\
$A_{2}$ & $\{0.4,0.2,0.3\}$ & $\{0.3,0.2,0.4\}$ & $\{0.9,0.0,0.1\}$ & $\{0.5,0.3,0.2\}$ \\
$A_{3}$ & $\{0.4,0.3,0.1\}$ & $\{0.5,0.1,0.3\}$ & $\{0.5,0.0,0.4\}$ & $\{0.6,0.2,0.2\}$ \\
$A_{4}$ & $\{0.6,0.1,0.2\}$ & $\{0.2,0.2,0.5\}$ & $\{0.4,0.3,0.2\}$ & $\{0.7,0.2,0.1\}$ \\
\hline
\end{tabular}

TABLE 5: Ranking results by different methods.

\begin{tabular}{lccc}
\hline The method & The ranking & The best & The worst \\
\hline Crossentropy & $A_{3}>A_{1}>A_{2}>A_{4}$ & $A_{3}$ & $A_{4}$ \\
TOPSIS & $A_{2}>A_{3}>A_{1}>A_{4}$ & $A_{2}$ & $A_{4}$ \\
The proposed method $(\lambda=0.5)$ & $A_{3}>A_{1}>A_{2}>A_{4}$ & $A_{3}$ & $A_{4}$ \\
\hline
\end{tabular}

neutrosophic sets which are given by the decision maker is shown in Table 4.

The above three methods are used to solve the decisionmaking problem, respectively, and the results of them are shown in Table 5.

From Table 5, it can be seen that the worst alternative is $A_{4}$ for all methods, the best one is $A_{3}$ for the proposed method and the crossentropy method in [21], while the best one is $A_{2}$ for the TOPSIS method in [17]. The ranking results are identical using the crossentropy method in [21] and the proposed method with $\lambda=0.5$. The result of the TOPSIS method differs from the other two methods.

The three methods have the different ideas to solve the MCDM with SVNSs. The crossentropy method in [21] calculates the crossentropy of each alternative to the positive ideal solution, which is used to rank alternatives. The TOPSIS method in [17] used the relative closeness coefficients to rank all alternative. The relative closeness coefficient is determined by the distances of an alternative from the positive ideal solution and the negative ideal solution. On the other hand, the two methods are not suitable for solving the MCDM problems with multivalue neutrosophic information, while the proposed method can be used to deal with single-value or multivalue neutrosophic information.

\section{Conclusion}

MVNSs are very useful in modeling the problems with uncertain, imprecise, incomplete, and inconsistent information, which widely exist in scientific and engineering cases. In this paper, based on the EDAS method in [49] and the proposed multivalued neutrosophic extended weighted average (MVNCWA) operator, we propose an extended EDAS method for decision-making problem described by MVNSs. In this method, the average alternative is calculated by aggregating the MVNNs under each criterion using the MVNCWA operator. Next, the positive distance and negative distance between each alternative and the average alternative under all the criteria are calculated. And then we revise the aggregation method of the positive distance and negative distance in [49] and permit the decision makers to adjust their proportions in calculating the comprehensive evaluations of alternatives according to their preferences. Two cases study are analyzed. The first one is to illustrate that the proposed method is stable for the adjusted parameter and is feasible and effective for solving MCDM problems with MVNSs by comparing with some existing methods. And the other is to compare the proposed method with two methods for SVNSs.

From the above analysis, it is obvious that the proposed method needs fewer data preprocessing and less calculation and is suitable to solve the decision-making problem with more criteria and alternatives. It can also be used to the situations in which the decision information is described by SVNSs, HFSs, or Dual HFSs, which are the special forms of MVNSs. Moreover, the decision makers' preference for the loss or gain of an alternative is taken into consideration in the proposed method, so as to get the most favourite solution.

In the future, we will investigate the decision-making method for MVNSs under criteria with different relationships and intend to study the social network-based methods for different formats of NSs.

\section{Data Availability}

All of the data used to support the findings of this study are included within the article. 


\section{Conflicts of Interest}

The authors declare that they have no conflicts of interest.

\section{Acknowledgments}

The paper was supported by the National Nature Science Foundation of China (nos. 71971190 and 71702087) and Shandong Province Social Science Planning Research Project (no. 18CHLJ34).

\section{References}

[1] L. A. Zadeh, "Fuzzy sets," Information and Control, vol. 8, no. 3, pp. 338-353, 1965.

[2] L. A. Zadeh, "The concept of a linguistic variable and its application to approximate reasoning-I," Information Sciences, vol. 8, no. 3, pp. 199-249, 1975.

[3] K. T. Atanassov, "Intuitionistic fuzzy sets," Fuzzy Sets and Systems, vol. 20, no. 1, pp. 87-96, 1986.

[4] K. Atanassov and G. Gargov, "Interval valued intuitionistic fuzzy sets," Fuzzy Sets and Systems, vol. 31, no. 3, pp. 343-349, 1989.

[5] V. Torra and Y. Narukawa, "On hesitant fuzzy sets and decision," in Proceedings of the 18th IEEE International Conference on Fuzzy Systems Jeju Island, pp. 1378-1382, Jeju Island, South Korea, August 2009.

[6] V. Torra, "Hesitant fuzzy sets," International Journal of Intelligent Systems, vol. 25, pp. 529-539, 2010.

[7] B. Farhadinia, "Hesitant fuzzy set lexicographical ordering and its application to multi-attribute decision making," Information Sciences, vol. 327, no. 10, pp. 233-245, 2016.

[8] P. Liu, "Some Hamacher aggregation operators based on the interval-valued intuitionistic fuzzy numbers and their application to group decision making," IEEE Transactions on Fuzzy Systems, vol. 22, no. 1, pp. 83-97, 2014.

[9] O. Sohaib, M. Naderpour, W. Hussain, and L. Martinez, "Cloud computing model selection for E-commerce enterprises using a new 2-tuple fuzzy linguistic decision-making method," Computers \& Industrial Engineering, vol. 132, pp. 47-58, 2019.

[10] F. Smarandache, Neutrosophy: Neutrosophic Probability, Set, and Logic, American Research Press, Rehoboth, DE, USA, 1998.

[11] F. Smarandache, "A unifying field in logics," in Neutrosophy: Neutrosophic Probability, Set and Logic, pp. 1-141, American Research Press, Rehoboth, DE, USA, 1999.

[12] F. Smarandache, Introduction to Neutrosophic Measure, Neutrosophic Integral, and Neutrosophic Probability, Education Publishing, Columbus, OH, USA, 2013.

[13] H. Wang, F. Smarandache, Y. Q. Zhang, and R. Sunderraman, "Single valued neutrosophic sets," Multispace and Multistructure, vol. 4, pp. 410-413, 2010.

[14] H. Wang, F. Smarandache, Y. Q. Zhang, and R. Sunderraman, "Single valued neutrosophic sets," in Proceedings of 10th International Conference on Fuzzy Theory and Technology, pp. 21-26, Salt Lake City, UT, USA, August 2005.

[15] P. D. Liu and Y. M. Wang, "Multiple attribute decisionmaking method based on single valued neutrosophic normalized weighted Bonferroni mean," Neural Computing and Applications, vol. 25, no. 7-8, pp. 2001-2010, 2014.

[16] P. D. Liu, Y. C. Chu, Y. W. Li, and Y. B. Chen, "Some generalized neutrosophic number Hamacher aggregation operators and their application to group decision making,"
International Journal of Fuzzy Systems, vol. 16, no. 2, pp. 242-255, 2014.

[17] P. P. Chi and P. D Liu, "An extended TOPSIS method for the multiple attribute decision making problems based on interval neutrosophic set," Neutrosophic Sets and Systems, vol. 1, pp. 1-8, 2013.

[18] P. Liu and L. Shi, “The generalized hybrid weighted average operator based on interval neutrosophic hesitant set and its application to multiple attribute decision making," Neural Computing and Applications, vol. 26, no. 2, pp. 457-471, 2015.

[19] P. Liu and G. Tang, "Some power generalized aggregation operators based on the interval neutrosophic sets and their application to decision making," Journal of Intelligent \& Fuzzy Systems, vol. 30, no. 5, pp. 2517-2528, 2016.

[20] P. Liu and Y. Wang, "Interval neutrosophic prioritized OWA operator and its application to multiple attribute decision making," Journal of Systems Science and Complexity, vol. 29, no. 3, pp. 681-697, 2016.

[21] J. Ye, "A multicriteria decision-making method using aggregation operators for simplified neutrosophic sets," Journal of Intelligent \& Fuzzy Systems, vol. 26, no. 5, pp. 2459-2466, 2014.

[22] J. Ye, "Multicriteria decision-making method using the correlation coefficient under single-valued neutrosophic environment," International Journal of General Systems, vol. 42, no. 4, pp. 386-394, 2013.

[23] Z.-P. Tian, J. Wang, J.-Q. Wang, and H.-Y. Zhang, "An improved MULTIMOORA approach for multi-criteria decision-making based on interdependent inputs of simplified neutrosophic linguistic information," Neural Computing and Applications, vol. 28, no. S1, pp. 585-597, 2017.

[24] S. Broumi, M. Talea, A. Bakali, and F. Smarandache, "Single valued neutrosophic graphs," Journal of New Theory, vol. 10, pp. 86-101, 2016.

[25] S. Broumi, A. Bakali, M. Talea, and F. Smarandach, "Isolated single valued neutrosophic graphs," Neutrosophic Sets and Systems, vol. 11, pp. 74-78, 2016.

[26] S. Broumi, M. Talea, A. Bakali, and F. Smarandache, "On bipolar single valued neutrosophic graphs," Journal of New Theory, vol. 11, pp. 84-102, 2016.

[27] S. Broumi, F. Smarandache, M. Talea, and A. Bakali, "An introduction to bipolar single valued neutrosophic graph theory," Applied Mechanics and Materials, vol. 841, pp. 184191, 2016.

[28] S. Broumi, M. Talea, A. Bakali, and F. Smarandache, "Interval valued neutrosophic graphs," Critical Review, vol. 12, pp. 533, 2016.

[29] S. Broumi, M. Talea, F. Smarandache, and A. Bakali, "Single valued neutrosophic graphs: degree, order and size," in Proceedings of the 2016 IEEE World Congress on Computational Intelligence, pp. 2444-2451, Vancouver, Canada, July 2016.

[30] H. V. Long, M. Ali, L. H. Son, M. Khan, and D. N. Tu, “A novel approach for fuzzy clustering based on neutrosophic association matrix," Computers \& Industrial Engineering, vol. 127, pp. 687-697, 2019.

[31] J. Ye, "Single valued neutrosophic minimum spanning tree and its clustering method," Journal of Intelligent Systems, vol. 23, no. 3, pp. 311-324, 2014.

[32] J. Ye, "Clustering methods using distance-based similarity measures of single-valued neutrosophic sets," Journal of Intelligent Systems, vol. 23, no. 4, pp. 379-389, 2014.

[33] A. Sengur and Y. Guo, "Color texture image segmentation based on neutrosophic set and wavelet transformation," 
Computer Vision and Image Understanding, vol. 115, no. 8, pp. 1134-1144, 2011.

[34] H. D. Cheng and Y. Guo, "A new neutrosophic approach to image thresholding," New Mathematics and Natural Computation, vol. 4, no. 3, pp. 291-308, 2008.

[35] Y. Guo and H. D. Cheng, "New neutrosophic approach to image segmentation," Pattern Recognition, vol. 42, no. 5, pp. 587-595, 2009.

[36] J. Ye, "Improved cosine similarity measures of simplified neutrosophic sets for medical diagnoses," Artificial Intelligence in Medicine, vol. 63, no. 3, pp. 171-179, 2015.

[37] J. Ye and J. Fu, "Multi-period medical diagnosis method using a single valued neutrosophic similarity measure based on tangent function," Computer Methods and Programs in Biomedicine, vol. 123, pp. 142-149, 2016.

[38] J. Ye, "Single-valued neutrosophic similarity measures based on cotangent function and their application in the fault diagnosis of steam turbine," Soft Computing, vol. 21, no. 3, pp. 817-825, 2017.

[39] D. Nagarajan, M. Lathamaheswari, S. Broumi, and J. Kavikumar, "A new perspective on traffic control management using triangular interval type-2 fuzzy sets and interval neutrosophic sets," Operations Research Perspectives, vol. 6, Article ID 100099, 2019.

[40] Z.-P. Tian, J. Wang, J.-Q. Wang, and H.-Y. Zhang, "Simplified neutrosophic linguistic multi-criteria group decision-making approach to green product development," Group Decision and Negotiation, vol. 26, no. 3, pp. 597-627, 2017.

[41] J. Wang, J.-Q. Wang, and H.-Y. Zhang, "A likelihood-based TODIM approach based on multi-hesitant fuzzy linguistic information for evaluation in logistics outsourcing," Computers \& Industrial Engineering, vol. 99, pp. 287-299, 2016.

[42] F. Smarandache and L. Vladareanu, "Applications of neutrosophic logic to robotics," Neutrosophic Theory and Its Applications, vol. 1, pp. 61-66, 2014.

[43] J.-Q. Wang and X. E. Li, “TODIM method with multi-valued neutrosophic sets," Control and Decision, vol. 30, no. 6, pp. 1139-1142, 2015.

[44] P. Liu, L. Zhang, X. Liu, and P. Wang, "Multi-valued neutrosophic number Bonferroni mean operators with their applications in multiple attribute group decision making," International Journal of Information Technology \& Decision Making, vol. 15, no. 5, pp. 1181-1210, 2016.

[45] J.-J. Peng and J.-Q. Wang, "Multi-valued neutrosophic sets and its application in multi-criteria decision-making problems," Neutrosophic Sets and Systems, vol. 10, pp. 3-17, 2016.

[46] J.-J. Peng, J.-Q. Wang, and X.-H. Wu, "An extension of the ELECTRE approach with multi-valued neutrosophic information," Neural Computing and Applications, vol. 28, no. S1, pp. 1011-1022, 2017.

[47] J.-J. Peng, J.-Q. Wang, and W.-E. Yang, "A multi-valued neutrosophic qualitative flexible approach based on likelihood for multi-criteria decision-making problems," International Journal of Systems Science, vol. 48, no. 2, pp. 425-435, 2017.

[48] P. Ji, H.-Y. Zhang, and J.-Q. Wang, "A projection-based TODIM method under multi-valued neutrosophic environments and its application in personnel selection," Neural Computing and Applications, vol. 29, no. 1, pp. 221-234, 2018.

[49] M. K. Ghorabaee, E. K. Zavadskas, L. Olfat et al., "Multicriteria inventory classification using a new method of evaluation based on distance from average solution (EDAS)," Informatica, vol. 26, no. 3, pp. 435-451, 2015.
[50] B. Zhu, Z. Xu, and M. Xia, "Dual hesitant fuzzy sets," Journal of Applied Mathematics, vol. 2012, Article ID 879629, 13 pages, 2012.

[51] C. Wei, N. Zhao, and X. Tang, "Operators and comparisons of hesitant fuzzy linguistic term sets," IEEE Transactions on Fuzzy Systems, vol. 22, no. 3, pp. 575-585, 2014. 